\title{
On the effect of insulator angle with respect to the cross arm on the distribution of lightning electric fields
}

\begin{abstract}
In this paper, the effect of insulator angle with respect to the cross arm on the electrical performance of a polymer insulator under lightning impulse voltage condition was studied and the results discussed accordingly. An insulator angle with the cross arm of other than $90^{\circ}$ is usually due to defects in the connections or being under pressure from the line angles and it can be an important issue for the stability of the line against lightning. The results show that the insulator angle can play an important role in increasing the electric fields around insulator and thus increase the chance of electrical breakdown of the insulator.
\end{abstract}

Keyword: Polymer insulator; Lightning; Electric fields 